\title{
Experimental and theoretical investigations of a rectangular grating structure for low-voltage traveling wave tube amplifiers
}

\author{
J. Joe, L. J. Louis, J. E. Scharer, J. H. Booske, and M. A. Basten \\ Electrical and Computer Engineering Department, University of Wisconsin, Madison, Wisconsin 53706
}

(Received 3 January 1997; accepted 19 February 1997)

\begin{abstract}
A periodic, rectangular grating slow-wave-structure is considered for forward and backward wave low-voltage $(\leqslant 10 \mathrm{kV})$ Ku-band traveling wave tube (TWT) amplifiers. For forward wave operation, it is required that the ratio of groove depth, $d$, to grating period, $p$, be large (i.e. $d / p \geqslant 5$ ) while small values of $d / p$ allow backward wave operation. For large $d / p$, skin effect losses in the grating slots are large and can substantially reduce the growth rate produced by the beam-slow wave interaction. Phase and amplitude measurements of the grating structures utilizing a slotted line and a fast Fourier transform (FFT) analysis have been carried out. The results show that the measured dispersion relations for both shallow $(d / p=0.446)$ and deep groove gratings $(d / p=7.43)$ agree very well with the theoretical dispersion relations. For amplifier experiments, a round "probe" beam (10 kV, $0.25 \mathrm{~A}, 1 \mathrm{~mm}$ radius) from a Litton Pierce electron gun (model M707) is utilized. The beam is confined by means of a $1 \mathrm{kG}$ focusing solenoidal magnetic field. Tuning curve and single particle backward wave gain measurements are presented and discussed. (C) 1997 American Institute of Physics. [S1070-664X(97)04507-2]
\end{abstract}

\section{INTRODUCTION}

A rectangular grating slow-wave structure has been utilized in several previous microwave oscillator experiments. ${ }^{1-3}$ These experiments employed electron beam energies $>100 \mathrm{kV}$. The configurations used were either open grating structures with a top mirror aligned parallel to the metal grating without sidewalls or an axial cavity reflector. In these configurations, the grating modes can be expanded in terms of pure transverse electric (TE) and transverse magnetic (TM) modes.

This paper presents an experimental investigation of a closed rectangular waveguide with grating structure at the lower boundary (Fig. 1) for low energy beam amplifier applications. We have previously published ${ }^{4}$ a theoretical analysis of the hybrid mode dispersion relation and the absolute and convective regions of wave growth for this configuration. This structure is sheet beam compatible. A sheet beam configuration ${ }^{5-7}$ is capable of carrying more beam current compared to that of a conventional round beam with less space charge because of its larger beam cross sectional area. Therefore, higher efficiency and higher power in a compact device can be obtained.

The normal modes for slow-wave-beam interaction in a grating-lined rectangular waveguide are expanded in terms of hybrid modes transverse electric to $x\left(T E_{x m n}\right),{ }^{8}$ where $x$ refers to the transverse dimension of the guide (the other set of hybrid modes, $T M_{x}$, operate at much higher frequencies). To understand the physics of low energy $(\mathrm{V} \leqslant 10 \mathrm{kV})$ beamamplifier interaction in a grating-lined rectangular waveguide, we have utilized two different configurations, a deep groove grating, and a shallow groove grating with a low energy "probe" round electron beam. Phase measurements for these two types of gratings are carried out to verify the theoretically predicted grating dispersion relations. This procedure is crucial to ensure the presence of the slow wave, with wavenumbers which satisfy the beam resonance condi- tion $\left(\omega=k_{z} v_{0}\right)$, that will interact with the beam at a particular frequency.

In Section II the theoretical dispersion relation for the grating structures is developed. In Section III, the phase measurement technique is described. The deep groove and shallow groove phase measurement results are discussed. The experimental configuration and gain measurement results are presented in Section IV. Skin effect loss calculations are also presented in this section. A summary section completes the paper.

\section{DISPERSION RELATION}

Consider a rectangular grating waveguide configuration shown in Fig. 1. The rectangular grating waveguide has a period $p$, groove width $s$, groove depth $d$, guide width $w$, and guide height $b$ from the grating surface to the top conductor. This configuration supports a set of hybrid modes called $\mathrm{TE}_{x} \cdot{ }^{9}$ This set of modes has all components of electric and magnetic fields except an $E_{x}$ component. The $\mathrm{TE}_{x 10}$ mode is the fundamental mode for this set of hybrid modes.

We have previously derived the dispersion relation for the $T E_{x}$ mode $^{4}$ which is given by

$$
D\left(\omega, k_{z}\right)=1-\nu_{x} p \tan \left(\nu_{x} d\right) \frac{s}{p_{n}} \sum_{=-\infty}^{\infty} \frac{\operatorname{sinc}^{2}\left(k_{z n} s / 2\right)}{\nu_{n} p \tanh \left(\nu_{n} b\right)}
$$

where $\nu_{x}^{2}=k_{0}^{2}-k_{x}^{2}, \nu_{n}^{2}=k_{z n}^{2}-\nu_{x}^{2}, \operatorname{sinc}\left(k_{z n} s / 2\right)=\left(\left[\sin \left(k_{z n} s /\right.\right.\right.$ 2) $] /\left[k_{z n} s / 2\right]$, and $k_{z n}$ is the Floquet spatial harmonic which satisfies the relation $k_{z n}=k_{z}+(2 \pi n / p)$.

To operate in the forward wave regime with a low voltage electron beam (i.e. $\leqslant 10 \mathrm{kV}$ ), it is necessary to utilize a grating with a large groove-depth-to-period ratio $\mathrm{d} / \mathrm{p} .{ }^{4} \mathrm{~A}$ shallow groove grating (small $d / p$ ) is utilized for backward wave regime operation with the same low voltage beam. The geometrical parameters for both types of these gratings selected for the purpose of our experimental investigations are 


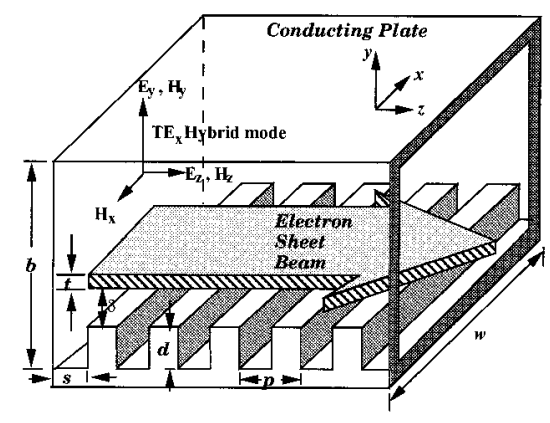

FIG. 1. Rectangular waveguide grating geometry.

listed in Table I. These parameters will be utilized throughout the paper to refer to shallow and deep groove gratings.

Utilizing the parameters in Table I and applying Eq.(1), one obtains the dispersion relations shown in Fig. 2. With the same $10 \mathrm{kV}$ beam, the resonance interaction point occurs in different regimes for the two gratings. For a deep groove grating, the resonance point occurs in the regime where the phase and group velocities of the slow wave propagate in the same direction. Conversely, for the shallow groove grating case, the resonance point occurs in the region where the phase and group velocities propagate in opposite directions.

\section{PHASE MEASUREMENTS}

\section{A. Experimental configuration}

Phase measurements for deep and shallow groove gratings were carried out to verify the theoretical grating dispersion relation. This procedure is crucial to verify the predicted mode and wavenumber that will interact with the beam at the resonance frequency. The scheme for the phase measurement experiment is shown in Fig. 3.

A slot was cut along an OFHC (oxygen-free-highconductivity) copper channel block. Four $8.89 \mathrm{~cm}$ segment combinations of smooth surface and grating pieces were inserted from below into the channel to form a smooth wall waveguide and a rectangular waveguide grating slow wave structure, respectively. A microwave signal from a synthesizer was connected to an SMA-to-Ku-band-waveguide coupler through a $-10 \mathrm{~dB}$ directional coupler. The signal from the output port of the directional coupler was launched into one end of the rectangular grating through a Ku-band waveguide. The signal from the $-10 \mathrm{~dB}$ directional coupler port

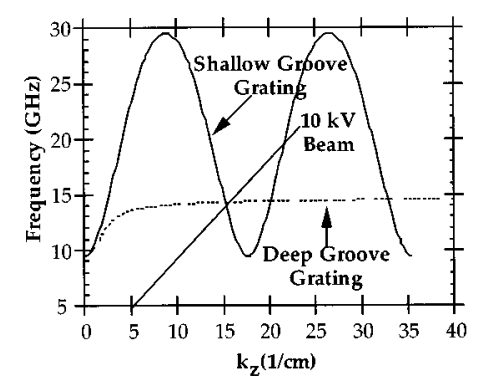

FIG. 2. $\mathrm{TE}_{x 10}$ dispersion curves for deep groove and shallow groove gratings.

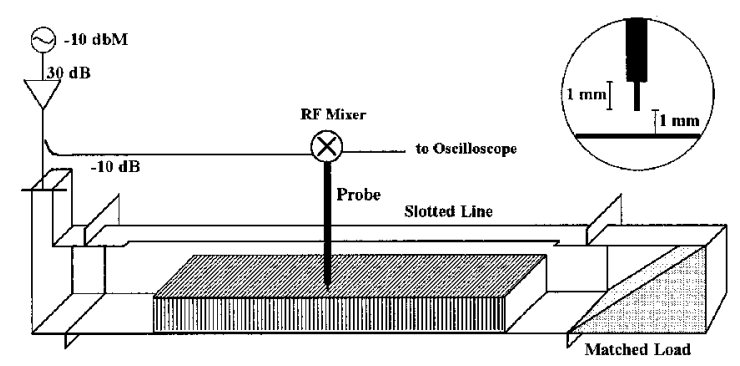

FIG. 3. Experimental set up for phase measurements.

was connected to the local oscillator (LO) port of a mixer and was used as the reference signal. The other end of the rectangular grating waveguide was terminated with a matched load. A dipole probe made out of microcoax was inserted into the slot of the channel block to probe the microwave signal which propagates in the rectangular grating waveguide. The probe signal was connected to the rf port of the mixer and mixed with the reference signal. A motor was utilized to drive the probe in linear motion over the slots to obtain the signal phase variation as a function of axial position in the waveguide. The dc output trace from the mixer's intermediate frequency (IF) port which contains the rectangular grating waveguide phase information was recorded and observed using a digitizing oscilloscope. A fast Fourier transform (FFT) of this trace was performed to obtain the $k_{z}$-spectrum of the wave propagating in the rectangulargrating-waveguide.

\section{B. Analysis}

Mathematically, the dc output trace from the mixer can be described as follows. Let the reference signal fed into the mixer's LO port be $V_{\mathrm{LO}} \cos \left(\omega t+\phi_{\mathrm{LO}}\right)$. $\phi_{\mathrm{LO}}$ is the phase shift from the directional coupler's $-10 \mathrm{~dB}$ port via a coaxial line to the mixer's LO port. The reflection coefficients due to the discontinuity at the Ku-band waveguide-grating interface at the input and output ends can be represented by $\Gamma_{1} \angle \theta_{1}$ and $\Gamma_{2} \angle \theta_{2}$, respectively. The reflection of the incident wave due to the probe can be written as $\Gamma_{p} \angle \theta_{p}$ where $\Gamma_{1}, \Gamma_{2}$, and $\Gamma_{p}$ are real numbers.

The fields inside the rectangular grating can be written in terms of forward wave and backward wave fields. The voltage representing these fields reads as

$$
\begin{aligned}
V_{F W} & =\sum_{n=-\infty}^{\infty} V_{n}^{+} \cos \left(\omega t+k_{z n} z+\phi_{n}^{+}\right), \\
V_{B W} & =\sum_{n=-\infty}^{\infty} V_{n}^{-} \cos \left(\omega t+k_{z n}(l-z)+\phi_{n}^{-}\right) .
\end{aligned}
$$

TABLE I. Grating parameters for deep and shallow groove gratings.

\begin{tabular}{lccccc}
\hline \hline \multicolumn{1}{c}{ Grating type } & $p(\mathrm{~mm})$ & $d(\mathrm{~mm})$ & $s(\mathrm{~mm})$ & $a(\mathrm{~mm})$ & $b(\mathrm{~mm})$ \\
\hline Shallow groove & 3.556 & 1.588 & 1.778 & 15.799 & 5.08 \\
Deep groove & 0.889 & 6.604 & 0.445 & 15.799 & 5.08 \\
\hline \hline
\end{tabular}


$V_{F W}$ and $V_{B W}$ are forward wave and backward wave voltages, $\phi_{n}^{+}$and $\phi_{n}^{-}$are phase shifts due to propagation path and intermediate components that the signal passes through from the synthesizer to the rectangular grating waveguide.

The total voltage detected by the dipole probe is given by

$$
\begin{aligned}
V_{p}= & \kappa \sum_{n=-\infty}^{\infty}\left\{V_{n}^{+} \cos \left(\omega t+k_{z n} z+\phi_{n}^{+}\right)+V_{n}^{-} \cos \left(\omega t+k_{z n}(l-z)+\phi_{n}^{-}\right)+\Gamma_{2} V_{n}^{+} \cos \left(\omega t+k_{z n}(l-z)+\phi_{n}^{+}+\theta_{2}\right)\right. \\
& +\Gamma_{1} V_{n}^{-} \cos \left(\omega t+k_{z n} z+\phi_{n}^{-}+\theta_{1}\right)+\Gamma_{1} \Gamma_{p} V_{n}^{+} \cos \left(\omega t+k_{z n} 3 z+\phi_{n}^{+}+\theta_{1}+\theta_{p}\right)+\Gamma_{2} \Gamma_{p} V_{n}^{-} \cos \left(\omega t+k_{z n} 3(l-z)+\phi_{n}^{-}\right. \\
& \left.\left.+\theta_{2}+\theta_{p}\right)+ \text { H.O.T. }\right\}
\end{aligned}
$$

$\kappa$ is the dipole probe coupling parameter, $\Gamma_{2} V_{n}^{+}$is the forward wave reflected by output end discontinuity, $\Gamma_{1} V_{n}^{-}$is the backward wave reflected by the input end discontinuity. $\Gamma_{p} \Gamma_{1} V_{n}^{+}$is the forward wave which is reflected back and forth by the probe and input end discontinuity, and $\Gamma_{p} \Gamma_{2} V_{n}^{-}$is the backward wave which is reflected back and forth by the probe and output end discontinuity. H.O.T. represents higher-order-terms which make a small contribution to Eq. (4).

The output from the mixer IF port is obtained by multiplying the mixer LO port signal to the mixer rf port signal which is equal to $V_{p}$. To first-order, the terms are given by $V_{\mathrm{IF}}=V_{\mathrm{IF}}^{\mathrm{dc}}+V_{\mathrm{IF}}^{\mathrm{ac}}$ where,

$$
\begin{aligned}
V_{\mathrm{IF}}^{\mathrm{dc}}= & \frac{\kappa V_{\mathrm{LO}}}{2} \sum_{n=-\infty}^{\infty}\left\{V_{n}^{+} \cos \left(k_{z n} z+\phi_{n}^{+}-\phi_{\mathrm{LO}}\right)+V_{n}^{-} \cos \left(k_{z n}(l-z)+\phi_{n}^{-}-\phi_{\mathrm{LO}}\right)\right. \\
& +\Gamma_{2} V_{n}^{+} \cos \left(k_{z n}(l-z)+\phi_{n}^{+}+\theta_{2}-\phi_{\mathrm{LO}}\right)+\Gamma_{1} V_{n}^{-} \cos \left(k_{z n} z+\phi_{n}^{-}+\theta_{1}-\phi_{\mathrm{LO}}\right) \\
& \left.+\Gamma_{1} \Gamma_{p} V_{n}^{+} \cos \left(k_{z n} 3 z+\phi_{n}^{+}+\theta_{1}+\theta_{p}-\phi_{\mathrm{LO}}\right)+\Gamma_{2} \Gamma_{p} V_{n}^{-} \cos \left(k_{z n} 3(l-z)+\phi_{n}^{-}+\theta_{2}+\theta_{p}-\phi_{\mathrm{LO}}\right)+\mathrm{H} \mathrm{O} . \mathrm{T}^{\mathrm{dc}} \cdot\right\}, \\
V_{\mathrm{IF}}^{\mathrm{ac}}= & \frac{\kappa V_{\mathrm{LO}}}{2} \sum_{n=-\infty}^{\infty}\left\{V_{n}^{+} \cos \left(2 \omega t+k_{z n} z+\phi_{n}^{+}+\phi_{\mathrm{LO}}\right)+V_{n}^{-} \cos \left(2 \omega t+k_{z n}(l-z)+\phi_{n}^{-}+\phi_{\mathrm{LO}}\right)\right. \\
& +\Gamma_{2} V_{n}^{+} \cos \left(2 \omega t+k_{z n}(l-z)+\phi_{n}^{+}+\theta_{2}+\phi_{\mathrm{LO}}\right)+\Gamma_{1} V_{n}^{-} \cos \left(2 \omega t+k_{z n} z+\phi_{n}^{-}+\theta_{1}+\phi_{\mathrm{LO}}\right) \\
& +\Gamma_{1} \Gamma_{p} V_{n}^{+} \cos \left(2 \omega t+k_{z n} 3 z+\phi_{n}^{+}+\theta_{1}+\theta_{p}+\phi_{\mathrm{LO}}\right)+\Gamma_{2} \Gamma_{p} V_{n}^{-} \cos \left(2 \omega t+k_{z n} 3(l-z)+\phi_{n}^{-}+\theta_{2}+\theta_{p}\right. \\
& \left.\left.+\phi_{\mathrm{LO}}\right)+\mathrm{H} \cdot \mathrm{O} \cdot \mathrm{T}^{\mathrm{ac}} .\right\} .
\end{aligned}
$$

$V_{\mathrm{IF}}^{\mathrm{dc}}$ is the dc component of IF port output and $V_{\mathrm{IF}}^{\mathrm{ac}}$ is the IF port output signal at a frequency twice that of the input microwave signal. The dc part is easily detected by oscilloscope.

By performing a Fourier transform with respect to $z$ on the dc component of the IF port output, the spectra of wavenumbers $k_{z}$ inside the rectangular grating waveguide can be obtained.

$$
\mathscr{T}_{\mathrm{IF}}^{\mathrm{dc}}\left(k_{z}\right)=\mathscr{F}\left\{V_{\mathrm{IF}}^{\mathrm{dc}}(z)\right\} .
$$

The results, if H.O.T ${ }^{\mathrm{dc}}$. are neglected, can be written as,

$$
\begin{aligned}
\mathscr{V}_{\mathrm{IF}}^{\mathrm{dc}}\left(k_{z}\right)= & \frac{\kappa V_{\mathrm{LO}} \pi}{2} \sum_{n=-\infty}^{\infty}\left\{\left[V_{n}^{+}+V_{n}^{-}+\Gamma_{2} V_{n}^{+}+\Gamma_{1} V_{n}^{-}\right]\right. \\
& \times \delta\left(k_{z} \pm k_{z n}\right)+\left[\Gamma_{1} \Gamma_{p} V_{n}^{+}+\Gamma_{2} \Gamma_{p} V_{n}^{-}\right] \\
& \left.\times \delta\left(k_{z} \pm 3 k_{z n}\right)\right\} .
\end{aligned}
$$

The spectrum of $\mathscr{T}_{\mathrm{IF}}^{\mathrm{dc}}\left(k_{z}\right)$ consists of $\pm k_{z n}, n=0,1,2 \ldots$, and $\pm 3 k_{z n}$. If H.O.T ${ }^{\mathrm{dc}}$. are included, $\pm(2 m+1) k_{z n}$ will be part of the solution to Eq. (8), where $m=2,3, \ldots$, . Note that the wavenumber spectra, during the Fourier transform calculations, are independent of all constant phase shifts components in Eq. (5) such as $\theta_{1}, \phi_{n}^{+}$. Reflections due to input and output end discontinuities add odd multiple wavenumber harmonics to the measurements.

\section{Results}

The dc output traces from the IF port on a long-timescale oscilloscope $(\approx 2 \mathrm{~min})$ were acquired utilizing a Lab View data acquisition software package. These data are then post-processed by utilizing the Matlab FFT package to obtain the wavenumber spectra information.

An illustrative example of a dc output trace and its FFT is shown in Figs. 4(a) and 4(b), respectively. This is a shallow groove case operating at $12.2 \mathrm{GHz}$. The primary signal is the fundamental spatial harmonic and the groove structure modulating the primary signal shown in Fig. 4(a) consists of high-order spatial harmonics. The third harmonic of the fundamental forward wave space harmonic is due to the small reflection of the signal by the probe which also modulates the primary signal such that the signal shape is no longer purely sinusoidal. The wavenumber spectrum of the longitudinal spatial trace is shown in Fig. 4(b) where it is easy to recognize the spectra of the fundamental spatial harmonic, third harmonic of the fundamental spatial harmonic, and the first-order spatial harmonic.

\section{Shallow groove configuration}

The shallow groove dispersion relation measurement agrees very well with the theoretical one. This is illustrated in Fig. 5. With the phase measurement technique, we are able to capture both the fundamental spatial harmonic and 


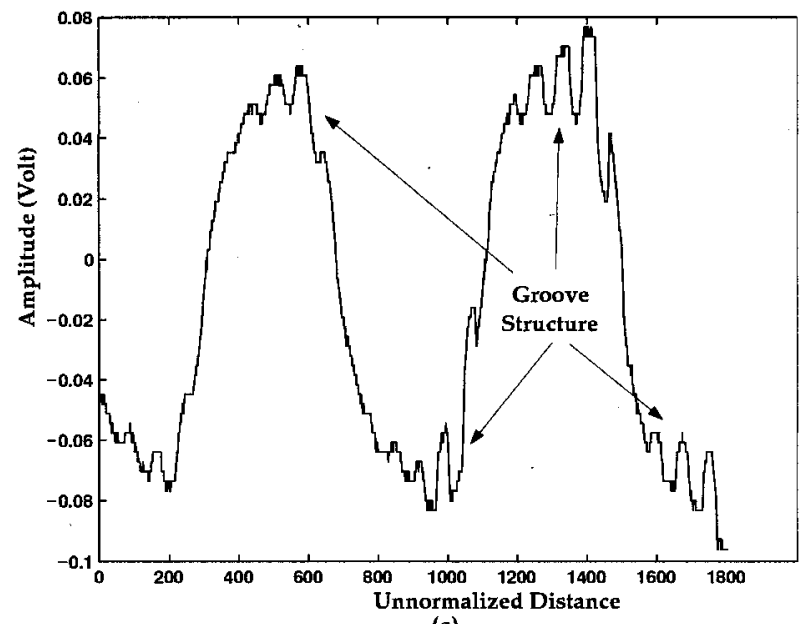

(a)

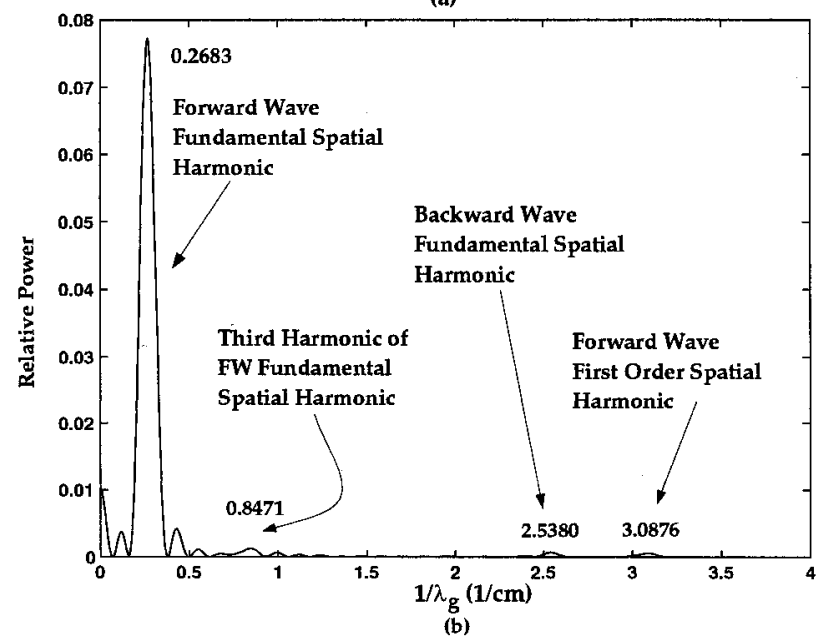

FIG. 4. Phase measurement illustrative example. (a) Spatial variation. (b) FFT of spatial variation.

the first-order spatial harmonic for the fundamental hybrid mode $\mathrm{TE}_{x 10}$. Note that along with the spatial harmonics, the third harmonic of the fundamental spatial harmonic is also present due to the probe's reflection. This is an artifact of the phase measurement system and would not be present in the absence of the probe.

Since the microwave system used for the phase measurements is calibrated up to $18 \mathrm{GHz}$ (limited primarily by the

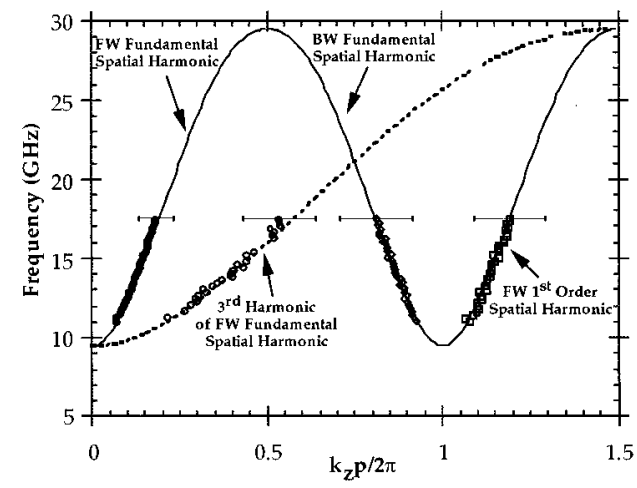

FIG. 5. Experimental results of shallow groove dispersion relation.

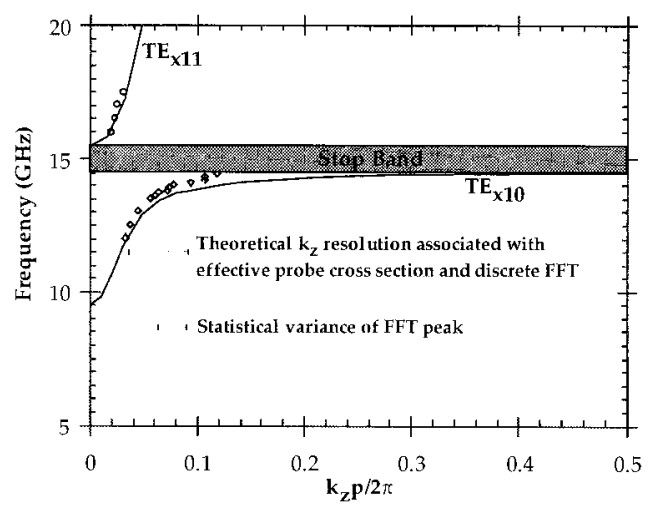

FIG. 6. Experimental results of deep groove dispersion relation.

mixer and the directional coupler), the phase measurements are discontinued at this frequency.

\section{Deep groove configuration}

The theoretical dispersion relation for a deep groove grating utilizing Eq. (1) and the measured dispersion relation using the phase measurement method described previously are compared in Fig. 6. Note that the third harmonic of the fundamental spatial harmonic due to probe reflections is no longer present. This is due to the large skin effect losses for the $\mathrm{TE}_{x 10}$ mode in a deep groove grating. The reflected signal from the probe becomes very weak after traveling three times the path length from the beginning of the grating to the probe. The third harmonic of the high order $\mathrm{TE}_{x 11}$ mode is still detectable (not shown in the figure) since the skin loss for this mode is small for the 15.5 to $17 \mathrm{GHz}$ frequency range.

The deep groove grating measured dispersion relation agrees very well with the calculated ones both for the $\mathrm{TE}_{x 10}$ and the $\mathrm{TE}_{x 11}$ modes. It is very difficult, however, to access the flat part of the $\mathrm{TE}_{x 10}$ dispersion curve $(14.0 \mathrm{GHz}$ $<$ frequency $\leqslant 14.5 \mathrm{GHz}$ ) because the loss due to skin effect absorption is large. The higher-order-spatial-harmonic power level is much smaller compared to the primary signal so that the FFT of $V_{\mathrm{IF}}^{\mathrm{dc}}$ no longer resolves the higher-order-spatialharmonic content.

\section{GAIN MEASUREMENTS}

\section{A. Experimental setup}

Figure 7 illustrates the schematic of the grating amplifier experiment. The electron beam is generated by an M707 Litton thermionic Pierce electron gun. The beam cross section is round and has a $1 \mathrm{~mm}$ radius. The perveance of the electron gun is $0.26 \mu$ pervs. The beam is focused and transported by a $1 \mathrm{kG}$ solenoidal dc magnetic field. The grating is placed inside the vacuum chamber. A pressure of $10^{-8}$ Torr inside the vacuum chamber is achieved during the experiment. The position of the grating with respect to electron beam can be mechanically adjusted from outside of vacuum chamber utilizing linear motion feedthrus. The pulse width of the beam is $30 \mu \mathrm{s}$. The beam is diverted at the downstream end to a OFHC copper beam dump. 


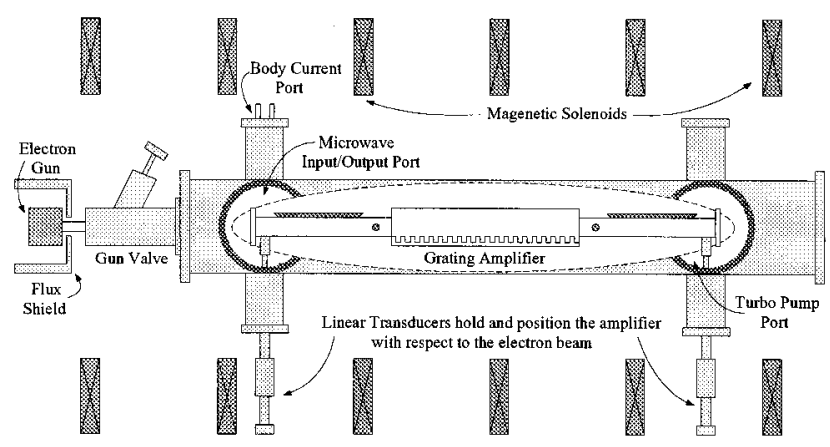

FIG. 7. Assembly for rectangular waveguide grating single particle gain experiment.

The microwave coupling and detection systems are illustrated in Fig. 8. Small loop antennas are transversely inserted at each end of the smooth wall rectangular waveguide for input and output $\mathrm{TE}_{z 10}$ mode coupling. The diameter of each loop is $2 \mathrm{~mm}$. Broadband, high vacuum compatible, silicon carbide attenuator fins are placed at both ends of rectangular waveguides. They provide excellent suppression $(-20 \mathrm{~dB})$ of unwanted end reflections and prevent the system from self-oscillation. For forward-wave-mode operation, the input signal is coupled into the grating through the upstream loop and detected by the downstream loop and vice versa for backward-wave-mode operation. The detected signal is fed into a narrowband tuned filter and amplified before being fed to a broadband crystal diode detector. The tuned filter is a Gaussian filter with a $20 \mathrm{MHz}$ bandwidth.

Both rectangular waveguides are electrically isolated from the grating structure utilizing high-vacuum-compatible thin mica insulators. This isolation allows the measurement of beam currents which intercept each section of the structure.

\section{B. Deep groove configuration for forward-wave-mode regime}

From the previous theoretical considerations, forwardwave-mode operation with a $10 \mathrm{kV}$ beam voltage is feasible for the deep groove configuration. The beam-slow wave resonance condition should occur at a frequency close to
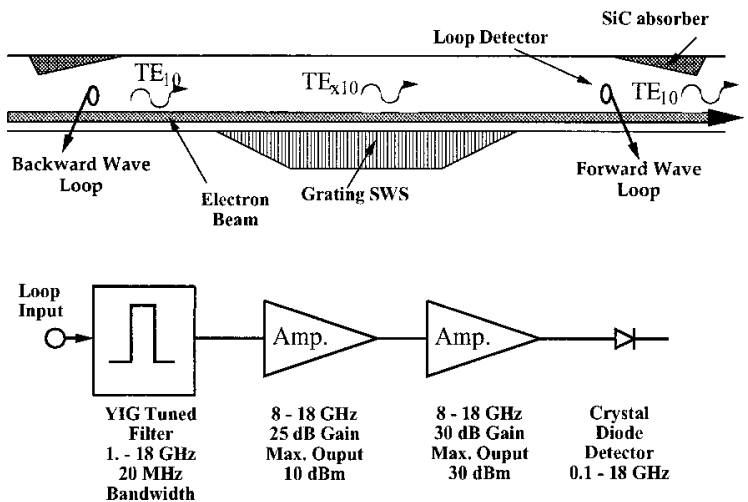

FIG. 8. Diagram for rectangular waveguide grating coupler and detection system.
4.2 GHz. However, the expected small signal gain was not bserved experimentally. This was determined to be due to arge skin effect losses in the deep groove grating structure.

Generally, the small skin loss for a rectangular waveuide grating can be obtained from the relation ${ }^{10}$

$$
\alpha=\frac{\oint_{\ell} \bar{H}_{\tau} \cdot \bar{H}_{\tau}^{*} d \ell}{2 \sigma \delta \int_{s} \operatorname{Re}\left(\bar{E}_{t} \times \bar{H}_{t}^{*}\right) d s},
$$

where $\bar{H}_{\tau}$ is magnetic field component tangential to the waveguide walls, $\sigma$ is the material conductivity, $\delta$ is the skin depth, and $\bar{E}_{t}$ and $\bar{H}_{t}$ are the transverse components of the electromagnetic field. For a rectangular waveguide grating, the skin loss calculation is simplified if it is carried out over one grating period $p$. The unit for $\alpha$ becomes Neper/period instead of Neper/meter. The skin effect loss for one period [Eq. (9)] becomes

$$
\alpha p=\frac{\int_{0}^{p} \oint_{\ell} \bar{H}_{\tau} \cdot \bar{H}_{\tau}^{*} d \ell d z}{2 \sigma \delta \frac{1}{p} \int_{0}^{p} \int_{s} \operatorname{Re}\left(\bar{E}_{t} \times \bar{H}_{t}^{*}\right) d s d z} .
$$

The expression for skin loss in Eqs. (9) and (10) only holds for weak damping, that is, when $\alpha / k_{z 0} \ll 1$. $^{11}$

The rectangular waveguide grating can be divided into two regions (see Fig. 1). $0 \leqslant y \leqslant d, 0 \leqslant z \leqslant s$ is region I and $d \leqslant y \leqslant b$ is region II. The magnetic field components in region I are

$$
\begin{aligned}
& H_{x}^{\mathrm{I}}=\frac{H_{0}}{j \omega \mu_{0}} \nu_{x}^{2} \sin \left(k_{x} x\right) \cos \left(\nu_{x} y\right), \\
& H_{y}^{I}=-\frac{H_{0}}{j \omega \mu_{0}} k_{x} \nu_{x} \cos \left(k_{x} x\right) \sin \left(\nu_{x} y\right),
\end{aligned}
$$

and a partial set of the electromagnetic field components in region II are

$$
\begin{aligned}
H_{x}^{\mathrm{II}}= & \frac{\nu_{x}^{2}}{j \omega \mu_{0}} \sin \left(k_{x} x\right) \sum_{n=-\infty}^{\infty} A_{n} \cosh \left(\nu_{n}(b-y)\right) e^{-j k_{z n} z}, \\
H_{y}^{\mathrm{II}}= & -\frac{k_{x}}{j \omega \mu_{0}} \cos \left(k_{x} x\right) \sum_{n=-\infty}^{\infty} A_{n} \nu_{n} \\
& \times \sinh \left(\nu_{n}(b-y)\right) e^{-j k_{z n} z} \\
H_{z}^{\mathrm{II}=} & -\frac{k_{x}}{j \omega \mu_{0}} \cos \left(k_{x} x\right) \sum_{n=-\infty}^{\infty} j k_{z n} A_{n} \\
& \times \cosh \left(\nu_{n}(b-y)\right) e^{-j k_{z n} z} \\
E_{y}^{\mathrm{II}}= & \sin \left(k_{x} x\right) \sum_{n=-\infty}^{\infty} j k_{z n} A_{n} \cosh \left(\nu_{n}(b-y)\right) e^{-j k_{z n} z}
\end{aligned}
$$

$A_{n}$ and $H_{0}$ are field coefficients. By matching $H_{x}^{\mathrm{I}}$ and $H_{x}^{\mathrm{II}}$ at $y=d, 0 \leqslant z \leqslant s, A_{n}$ can be expressed in terms of $H_{0}$ as

$$
A_{n}=\frac{\nu_{x} s H_{0} \sin \left(\nu_{x} d\right) \operatorname{sinc}\left(k_{z n} s / 2\right) e^{j k_{z n} s / 2}}{\nu_{n} d \sinh \left(\nu_{n}(b-d)\right)} .
$$




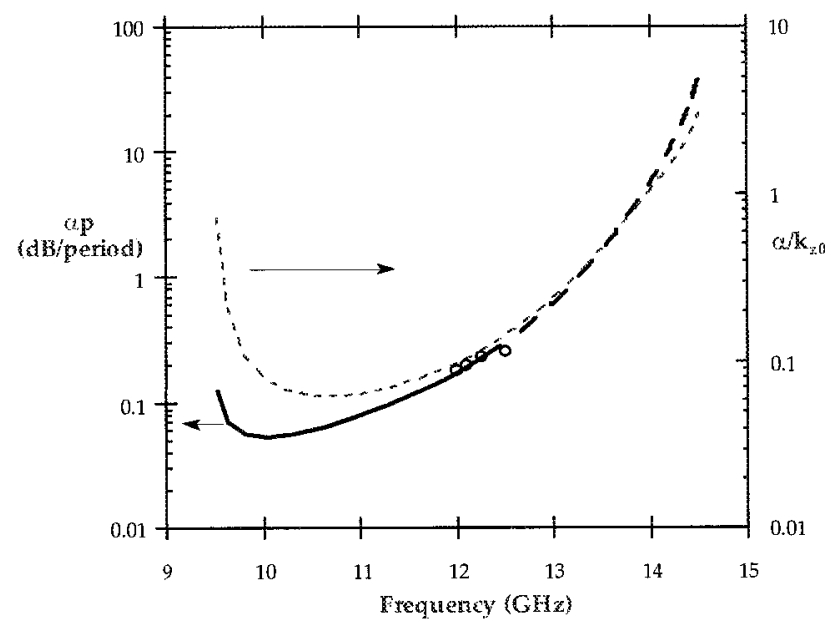

FIG. 9. The calculated and experimental data for skin loss and the ratio of $\alpha / k_{z 0}$ for the deep groove grating.

By evaluating all the magnetic fields at the waveguide and groove walls and carrying out the integral in the numerator of Eq. (10), evaluating the denominator of Eq. (10) using $E_{y}^{\mathrm{II}}$ and $H_{x}^{\mathrm{II}}$ and substituting the results utilizing the relation in Eq. (13), one obtains

$$
\alpha p=\frac{\zeta+\psi}{2 \sigma \delta P},
$$

where

$$
\begin{aligned}
\zeta= & \frac{1}{\left(\omega \mu_{0}\right)^{2} \sin ^{2}\left(\nu_{x} d\right)}\left\{\frac{a s \nu_{x}^{2} \sin ^{2}\left(\nu_{x} d\right)}{2}+k_{x}^{2} s\right. \\
& \left.\times\left[d-\frac{\sin \left(2 \nu_{x} d\right)}{2 \nu_{x}}\right]+\frac{a d k_{0}^{2}}{2}+a\left[\nu_{x}^{2}-k_{x}^{2}\right] \frac{\sin \left(2 \nu_{x} d\right)}{4 \nu_{x}}\right\}, \\
\psi= & \frac{p s^{2}}{\left(\omega \mu_{0} d\right)^{2}} \sum_{n=-\infty}^{\infty} \frac{\operatorname{sinc}^{2}\left(k_{z n} s / 2\right)}{\nu_{n}^{2} \sinh ^{2}\left(\nu_{n}(b-d)\right)} \\
& \times\left\{\frac{a}{2}\left[\cosh ^{2}\left(\nu_{n}(b-d)\right)+1\right]\left[\nu_{x}^{4}+k_{x}^{2} k_{z n}^{2}\right]\right. \\
& +k_{x}^{2} \frac{\sinh \left(2 \nu_{n}(b-d)\right)}{2 \nu_{n}}\left[\nu_{n}^{2}+k_{z n}^{2}\right] \\
& \left.+k_{x}^{2}(b-d)\left[k_{z n}^{2}-\nu_{n}^{2}\right]\right\}, \\
P= & \frac{a s^{2} \nu_{x}^{2}}{4 \omega \mu_{0} d^{2}} \sum_{n=-\infty}^{\infty} \frac{k_{z n} \operatorname{sinc}^{2}\left(k_{z n} s / 2\right)}{\nu_{n}^{2} \sinh ^{2}\left(\nu_{n}(b-d)\right)} \\
& \times\left\{(b-d)+\frac{\sinh ^{2}\left(2 \nu_{n}(b-d)\right)}{2 \nu_{n}}\right\}
\end{aligned}
$$

$\zeta$ and $\psi$ are the terms that describe the skin effect loss in regions I and II, respectively.

Figures 9 and 10 illustrate the calculated skin effect loss and the ratio of $\alpha / k_{z 0}$ for deep and shallow groove gratings, respectively (the parameters for both gratings are shown on Table I). For both cases, the skin effect losses are high near

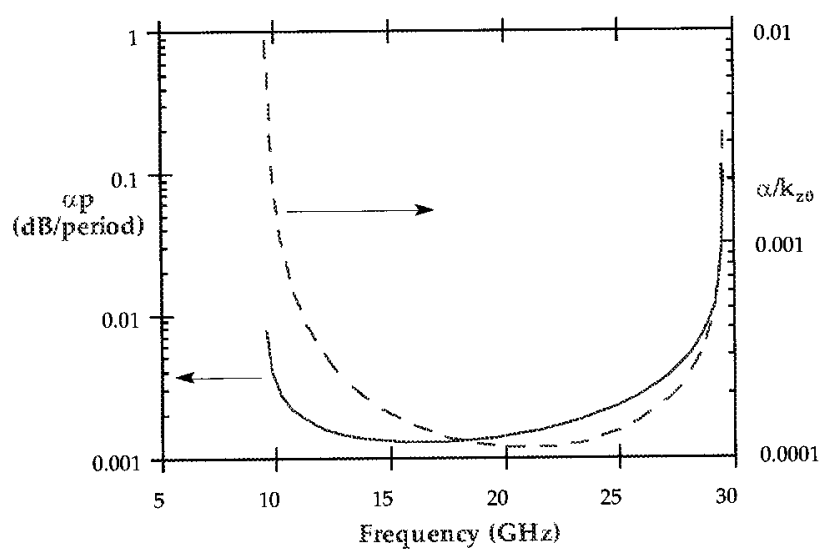

FIG. 10. The calculated skin loss and the ratio of $\alpha / k_{z 0}$ for the shallow groove grating.

the cutoff and stop-band frequencies where the group velocities are close to zero. The loss near the cutoff frequency for the $\mathrm{TE}_{x 10}$ mode is on the order of that corresponding to the $\mathrm{TE}_{z 10}$ mode. Note that in Fig. 9, Eq. (14) is no longer rigorously valid for frequencies above $12.25 \mathrm{GHz}$ (for the $\mathrm{TE}_{x 10}$ mode) since the ratio of $\alpha / k_{z 0}$ is no longer small (i.e. $\left.\alpha / k_{z 0}<0.1\right)$. By comparing Figs. 9 and 10, the skin effect loss for the deep groove grating is shown to be much larger than that corresponding to the shallow groove grating at the desired Ku-band operation frequency of $12-16 \mathrm{GHz}$ (this is why we are able to observe small signal gain for a shallow groove grating which is discussed in the next subsection). The dominant loss for the deep groove grating comes from the wall surface areas at $z=0$ and $z=s$ in region I (see Fig. 1). These surface areas for the shallow groove grating are only about one-fifth of those for the deep groove grating. Therefore, the skin effect loss can be reduced by decreasing the groove depth, $d$, of the grating. The calculated skin effect losses for the deep groove grating agree quite well with the network analyzer measurements for frequencies below 12.25 $\mathrm{GHz}$ as shown in Fig. 9. For example, at $12 \mathrm{GHz}$, the theoretically predicted loss is $-20 \mathrm{~dB}$ for a 100 grating period. The measured skin effect loss is $-18 \mathrm{~dB}$. Note that at this frequency, the calculated loss is marginally valid since $\alpha / k_{z 0} \approx 0.1$.

Figure 11 plots the shallow groove grating skin loss per

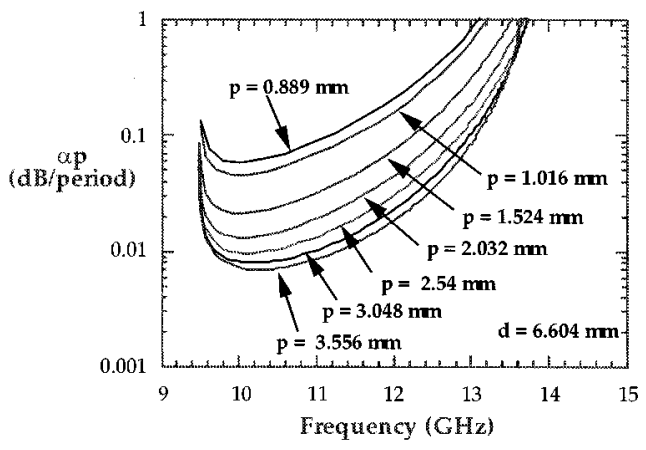

FIG. 11. Shallow groove grating skin loss per period versus normalized frequency for various groove depths. 


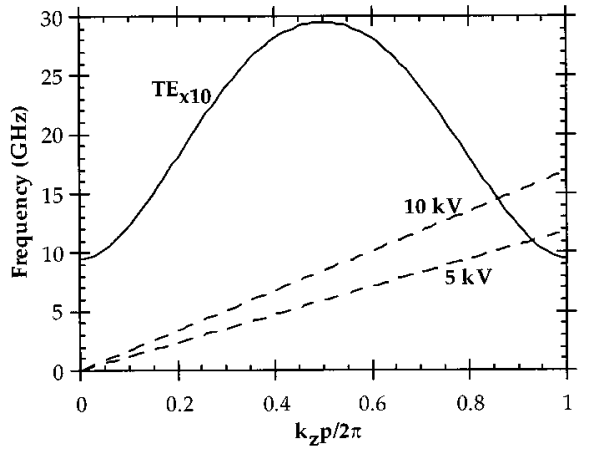

FIG. 12. Illustration of shallow groove backward wave mode tunability frequency range from 5 to $10 \mathrm{kV}$.

period versus frequency for various groove periods and a fixed groove depth $(d=6.604 \mathrm{~mm})$ and a fixed $s / p$ ratio $(s / p=0.5)$. As the grating period increases, the minimum value of the skin loss decreases. Figure 11 suggests that in order to keep the skin loss below a particular value at a given frequency, one should not exceed a certain $d / p$. For example, at $12 \mathrm{GHz}$, in order to keep the skin loss below 0.03 $\mathrm{dB} /$ period, one should not exceed $d / p=2.6$. In this case, the groove period must be larger than 100 mils or $2.54 \mathrm{~mm}$.

\section{Shallow groove configuration for backward wave amplification}

In the previous subsection, it has been shown that the shallow groove grating has low skin effect losses at the desired operation frequencies $(12-16 \mathrm{GHz})$. Therefore, we should expect to observe small-signal-gain in the backwardwave-mode regime for this grating configuration. From the dispersion relation shown in Fig. 12, it is obvious that this configuration has wideband tunability.

To experimentally verify this, we utilized the experimental configuration shown in Fig. 8. No input signal was coupled into the grating. The peak, incoherent spontaneous emission frequency response for various beam voltages were detected through the upstream loop. The results are plotted in Fig. 13 and are in good agreement with the theoretical tuning curve. This configuration is tunable from 10 to $14 \mathrm{GHz}$ with low beam energies, in this case from 4 to $9 \mathrm{kV}$.

Using a similar experimental arrangement, the microwave excitation was coupled into the grating through the

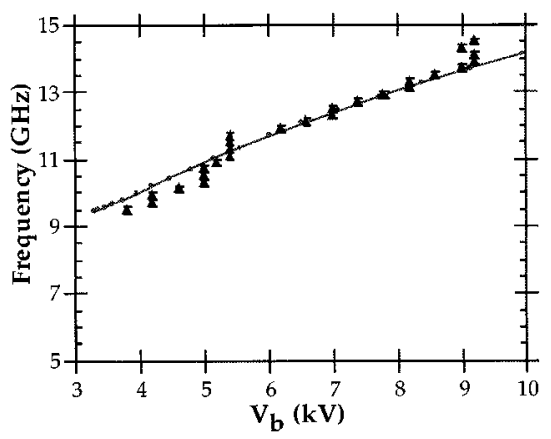

FIG. 13. Shallow groove experimental tuning curve compared to theoretical one.

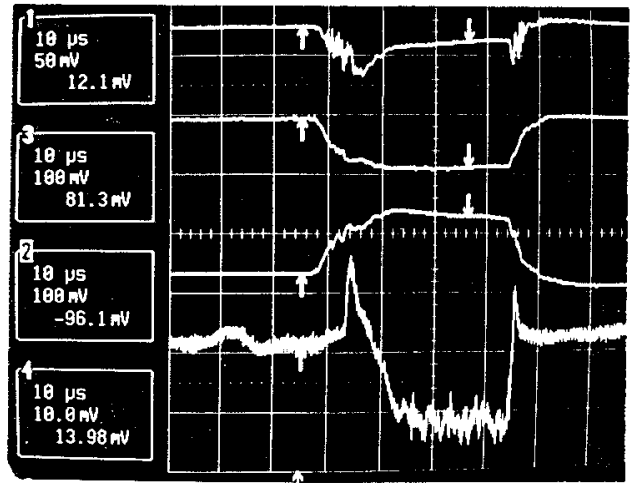

FIG. 14. A typical gain/absorption trace during single particle gain measurements.

downstream loop and small signal gain for the backward wave was detected at the upstream loop. Typical scope traces observed during small signal gain measurements are illustrated in Fig. 14. These traces were taken when the beam was grazing over the grating surface of two $8.89 \mathrm{~cm}$ long shallow groove grating segments. Trace 1 is the beam current collected by the upstream smooth waveguide section. Trace 3 is the beam current collected by the grating surface and trace 2 is the beam current collected by the downstream smooth waveguide section. Trace 4 is the time average microwave signal detected by a negative voltage diode detector. The center frequency of the tuned-band-pass filter is tuned to 13.42 GHz. The transient response in traces 1, 2, and 3 are associated with the rise and fall at the electron gun cathode voltage pulse. The rise-time is about $10 \mu \mathrm{s}$.

During the transient period, the beam voltage increases to finally reach a steady-state voltage, in this case $9.4 \mathrm{kV}$. Since the transient provides a voltage sweep, trace 4 should exhibit microwave energy absorption and gain at this frequency during the rise time. This is indeed the case. Trace 4 in Fig. 14 starts with a rise above the zero reference line (a decreased signal for the negative response voltage diode) and monotically becomes more negative until it reaches about 14 $\mathrm{mV}$ below the zero reference line. The curve above the zero reference line corresponds to microwave energy absorption by the electron beam and that below the zero reference line corresponds to microwave energy gain.

Classic experimental antisymmetric gain curves for 13.0 and $13.42 \mathrm{GHz}$ are shown in Fig. 15. Normalized gain in this

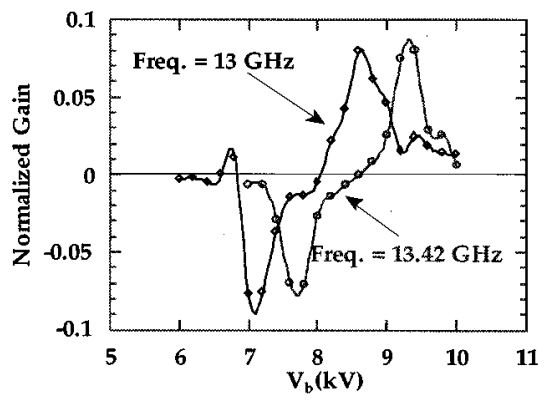

FIG. 15. Shallow groove single particle gain curves for 13 and $13.42 \mathrm{GHz}$. 


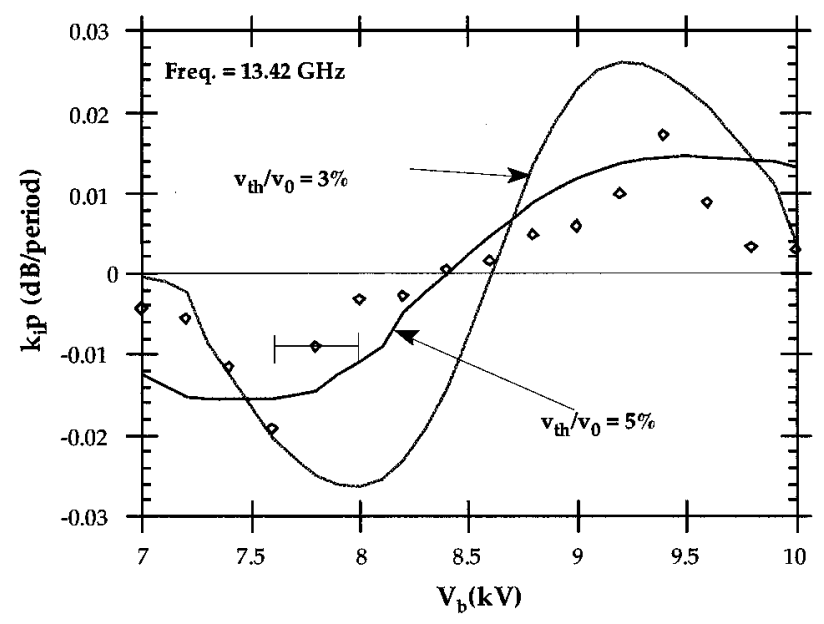

FIG. 16. Comparison between theoretical and experimental growth rates at $13.42 \mathrm{GHz}$.

figure is defined as $\left(P_{\text {out }}-P_{\text {in }}\right) / P_{\text {in }}$. Every experimental point illustrated in this figure is obtained by averaging 50 waveforms whose shapes are similar to that of trace 4 shown in Fig. 14. Note that when $\omega / v_{e}=k_{z 0}$, the normalized gain is actually close to zero. For the 13.0 and $13.42 \mathrm{GHz}$ cases, this corresponds to 8 and $8.5 \mathrm{kV}$ beam energies, respectively. For both cases, optimum gain occurs when the beam velocity is slightly greater than that at resonance, and the peak absorption occurs when the beam velocity is slightly less than that at resonance. In other words, the slow wave gains energy when it interacts with the slow space charge wave and loses energy when it interacts with the fast space charge wave. ${ }^{4}$

The theoretical growth rate ${ }^{4}$ per groove period at 13.42 $\mathrm{GHz}$ for various axial velocity spreads has been compared with the experimental results. Three $8.89 \mathrm{~cm}$ segments of shallow groove gratings are utilized. The beam density used for calculations was $32 \mathrm{~A} / \mathrm{cm}^{2}$ which is approximately equal to the beam density for the M707 electron gun at $10 \mathrm{kV}$. The comparison is illustrated in Fig. 16. Each experimental data point represented in the figure is obtained by averaging 50 shots. Let $\Delta V_{b}$ be the beam voltage difference between $\left.V_{b}\right|_{\text {max.growth }}$ and $\left.V_{b}\right|_{\text {max.absorption. The figure shows that as }}$ $v_{t h} / v_{0}$ increases, $\Delta V_{b}$ also increases. The experimental $\Delta V_{b}$ is close to that corresponding to a $5 \%$ axial velocity spread. Also, the experimental maximum growth rate per period is very close to the $5 \%$ axial velocity spread maximum growth rate per period. This is consistent with the previous and recent beam spread measurements utilizing an energy analyzer and Faraday cup. ${ }^{12}$ The beam spread is due to various factors associated with magnetic beam optics including a sensitivity to alignment of the gun magnetic flux shield. During the experiment, we also searched for absolute instabilities by looking for gain without an input signal in the 12.8 to $14.2 \mathrm{GHz}$ frequency range. However, no coherent absolute instabilities were observed. This is consistent with a $5 \%$ axial velocity spread. ${ }^{4}$

The growth rates calculated in Fig. 16 are for a sheetbeam configuration. The experimental growth rate obtained, however, is for a pencil-beam. The pencil beam growth rate

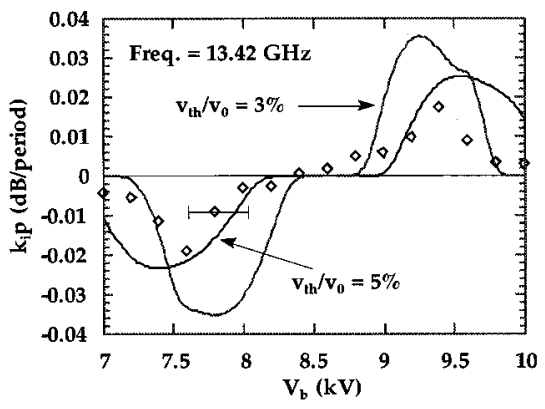

FIG. 17. Comparison between experimental and convoluted theoretical growth rates at $13.42 \mathrm{GHz}$.

differs from that of the sheet beam by a filling factor to the one-third power, $F^{1 / 3},{ }^{13}$ in the high-gain-compton regime (this becomes $F^{1 / 2}$ in the Raman regime and $F$ in the lowgain-Compton regime ${ }^{13}$ ).

For a $0.5 \mathrm{~mm}$ beam radius, $F^{1 / 3}$ is approximately equal to 0.5 . Therefore, the sheet-beam growth rate would be about twice the pencil-beam growth rate in the linear regime (it would be three times larger in the Raman regime and about ten times larger in the low-gain-Compton regime).

Figure 17 illustrates the comparison between experimental growth rate and the theoretical growth rate frequency response for a 5\% axial velocity spread which is convoluted with a $70 \mathrm{MHz}$ bandwidth Gaussian band-pass filter. The theoretical growth rate shown in this figure includes the high-gain-Compton regime filling factor and is compared with the experimental growth rate. The convolution changes the theoretical growth rate curve from a parabolic shape to a bell shape which is closer to that corresponding to the experimental curve. The experimental growth rate is little smaller than the theoretical growth rate. This is due to the fact that the device operation is in transition between the low-gain-Compton regime and high-gain-Compton regime. The device must satisfy the condition $Q l^{3} \ll 1$ and $Q^{1 / 3} \gg \omega_{p} / v_{0}{ }^{14}$ for operation in low gain and high-gainCompton regimes, respectively. $Q$ is a parameter which incorporates the coupling parameter $\kappa$, and the beam plasma frequency and axial velocity $Q=\kappa\left(\omega_{p} / V_{o z}\right)^{2}$. For our case, $Q=35 \mathrm{~cm}^{-3}, \omega_{p} / v_{0}=1.7 \mathrm{~cm}^{-1}$ and $l=27 \mathrm{~cm}$. Hence, the device marginally satisfies the high-gain-Compton regime. Corrections to the beam filling factor account for the factor of two difference between the theory and experiment.

Normalized gain versus the beam position with respect to the grating surface is plotted in Fig. 18. The gain is maximized when the beam is just grazing the grating surface and decays exponentially as the beam is moved away from the grating. This is expected since the magnitude of the $E_{z}$ field component of the slow wave which interacts with the beam decays exponentially away from the grating surface. The power decay rate is proportional to $\exp ^{-2 \nu_{0} y}$, where $y$ is the distance of the beam with respect to grating surface and $\nu_{0}^{2}=k_{z 0}^{2}-k_{0}^{2}+k_{x}^{2}$. At $8.8 \mathrm{kV}$, the frequency resonates at $13.54 \mathrm{GHz}$ and at $k_{z 0}=15.48 \mathrm{~cm}^{-1}$. The theoretically predicted $e$-folding distance for the coupling reduction due to beam-grating spacing is $1.1 \mathrm{~mm}$ which agrees very well with the experimental result shown in Fig. 18. When the beam is 


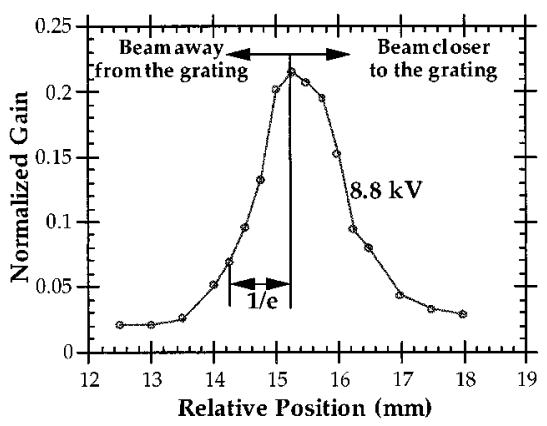

FIG. 18. Shallow groove small signal gain versus beam position.

moved closer to the grating than the grazing incidence location, the gain is also reduced since many of the beam electrons intercept the grating surface and are no longer available to interact with the slow wave.

\section{SUMMARY}

We have carried out an analysis and experimental investigation for shallow and deep groove grating slow wave amplifiers. The phase measurement results for both shallow and deep groove gratings match very well with the analytic dispersion relation. The experimental tuning curve and smallsignal gain have also been presented for the shallow groove grating configuration. The experimental tuning curve agrees well with the theoretical one. The small-signal gain measurement suggests the device is operating marginally in the highgain-Compton regime. For the deep groove grating, however, no small signal gain is observed due to large skin effect losses at the desired operation frequency.

\section{ACKNOWLEDGMENTS}

This work was supported by the Office of Naval Research (Grant No. N000 14-95-1-G022), the Department of Defense Vacuum Electronics Initiative as managed by the Air Force Office of Scientific Research (Grant No. AFOSR91-0381), and by a National Science Foundation Presidential Young Investigator Award (ECS-9057675).

${ }^{1}$ F. S. Rusin and G. D. Bogomolov, Proc. IEEE 57, 720 (1969).

${ }^{2}$ E. M. Marshall, P. M. Phillips, and J. E. Walsh, IEEE Trans. Plasma Sci. 16, 199 (1988).

${ }^{3}$ G. Dohler, D. Gallagher, F. Scafuri, and D. Wortman, Proceedings International Electron Devices Meeting (IEEE) (Institute of Electrical and Electronics Engineers, New York, 1987), pp. 303-306.

${ }^{4}$ J. Joe, J. Scharer, J. Booske, and B. McVey, Phys. Plasmas 1, 176 (1994).

${ }^{5}$ J. H. Booske, A. H. Kumbasar, and M. A. Basten, Phys. Rev. Lett. 71, 3979 (1993).

${ }^{6}$ J. H. Booske, M. A. Basten, A. H. Kumbasar, T. M. Antonsen, S. W. Bidwell, Y. Carmel, W. W. Destler, V. L. Granatstein, and D. J. Radacket, Phys. Plasmas 1, 1714 (1994).

${ }^{7}$ M. A. Basten, J. H. Booske, and J. Anderson, IEEE Trans. Plasma Sci. 22 960 (1994).

${ }^{8}$ R. F. Harrington, Time-harmonic Electromagnetic Fields (McGraw-Hill, New York, 1961), pp. 153-154.

${ }^{9}$ B. D. McVey, M. A. Basten, J. H. Booske, J. Joe, and J. E. Scharer, IEEE Trans. Microwave Theory and Techniques 42, 995 (1994).

${ }^{10} \mathrm{Om}$ P. Gandhi, Microwave Engineering and Applications (Pergamon, New York, 1981), pp. 23-24.

${ }^{11} \mathrm{P}$. Bhartia and I. J. Bahl, Millimeter Wave Engineering and Applications (Wiley, New York, 1984), p. 190.

${ }^{12}$ H. Kirolous (private communications, 1994).

${ }^{13}$ C. W. Roberson and P. Sprangle, Phys. Fluids B 1, 9 (1989).

${ }^{14}$ A. Gover and P. Sprangle, IEEE J. Quantum Electron. QE-17, 1196 (1981).

${ }^{15}$ J. Joe, Ph.D. thesis, University of Wisconsin-Madison, 1996.

${ }^{16}$ G. S. Park, S. Y. Park, R. H. Kyser, C. M. Armstrong, A. K. Ganguly, and R. K. Parker, IEEE Trans. Plasma Sci. 22, 536 (1994). 